\title{
CPTAC Terminology
}

National Cancer Institute

\section{Source}

National Cancer Institute. CPTAC Terminology. NCI Thesaurus. Code C156952.

Terminology used in support of the data collection efforts of the Clinical Proteomic

Tumor Analysis Consortium (CPTAC). 